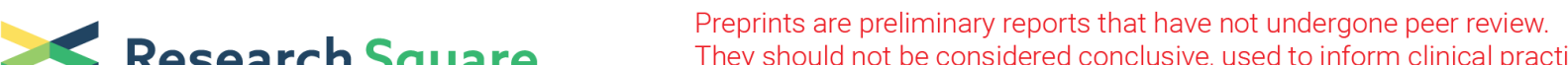 $\begin{array}{ll}\text { Research Square } & \text { They should not be considered conclusive, used to inform clinical practice, } \\ \text { or referenced by the media as validated information. }\end{array}$
}

\section{Nanoparticles Delivering Antagomir-483-5p to Bone Marrow Mesenchymal Stem Cells Prevent Osteoporosis by Increasing Bone Formation}

\section{Yue Zhou}

Hainan Medical College: Hainan Medical University

Hao Jia

Hainan Medical College: Hainan Medical University

Aihua $\mathrm{Hu}$

Hainan Medical College: Hainan Medical University

Rangru Liu

Hainan Medical College: Hainan Medical University

Xiangzhou Zeng

Hainan Medical College: Hainan Medical University

Hua Wang ( $\nabla$ wanghua8031@126.com )

Hainan Medical College: Hainan Medical University https://orcid.org/0000-0002-7215-4119

\section{Research}

Keywords: BMSC osteoblastic differentiation, miR-483-5p, osteoporosis, targeted drug delivery platform, MAPK1, Smad5

Posted Date: September 20th, 2021

DOl: https://doi.org/10.21203/rs.3.rs-882695/v1

License: (c) (i) This work is licensed under a Creative Commons Attribution 4.0 International License. Read Full License 


\section{Abstract}

Background: Promoting bone marrow mesenchymal stem cell (BMSC) osteoblastic differentiation is a promising therapeutic strategy for osteoporosis (OP). The present study demonstrates that miR-483-5p inhibits the osteogenic differentiation of BMSCs. Therefore, selectively delivering the nanoparticles carrying antagomir-483-5p (miR-483-5p inhibitor) to BMSCs is expected to become an effective treatment drug for OP.

Methods: Real-time PCR assays were used to analyse miR-483-5p, ALP and Bglap levels in BMSCs of ovariectomized and aged osteoporotic mice. To selectively and efficiently deliver antagomir-483-5p to BMSCs in vivo, immunoglobulin $\mathrm{G}$ and poloxamer-188 were used to encapsulate the functional small molecules, and BMSC-targeting aptamer was employed to confirm the direction of the nanoparticles. Luciferase assays were used to determine the target genes of miR-483-5p. Western blot assays and immunohistochemistry staining were used to detect the targets in vitro and vivo.

Results: miR-483-5p levels were increased in BMSCs of ovariectomized and aged osteoporotic mice. Inhibition of miR-483-5p levels in BMSCs by antagomir-483-5p in vitro promoted the expression of bone formation markers, such as ALP and Bglap. The FAM-BMSC-aptamer-nanoparticles carrying antagomir483-5p were taken up by BMSCs, resulting in stimulation of BMSC osteoblastic differentiation in vitro and osteoporosis prevention in vivo. Furthermore, our research demonstrated that mitogen-activated protein kinase 1 (MAPK1) and SMAD family member 5 (Smad5) were direct targets of miR-483-5p in regulating BMSC osteoblastic differentiation and osteoporosis pathological processes.

Conclusions: The important therapeutic role of FAM-BMSC-aptamer-nanoparticles carrying antagomir483-5p in osteoporosis was established in our study. These nanoparticles are novel candidate for the clinical prevention and treatment of osteoporosis. The optimized targeted drug delivery platform for small molecules will provide new ideas for the treatment of clinical diseases.

\section{Background}

Osteoporosis (OP) is a multifactorial disorder characterized by low bone mineral density and fragility fractures. Approximately nine million fractures every year worldwide are associated with OP. In the developed world, approximately $9-38 \%$ of females and $2-8 \%$ of males are affected by OP, and the incidence rate increases with age, especially for seniors over 60[1]. Most cases of OP is often related to either postmenopausal estrogen loss or age-related deterioration of skeletal microarchitecture, both of which are due to uncoupling in the bone remodeling unit. Bone remodeling, tightly couples with bone formation performed by osteoblasts and bone resorption facilitated by osteoclasts[2]. Importantly, disruption of homeostasis, such as inadequate bone formation or enhanced bone resorption, has a fundamental role in the pathogenesis of OP. Over the past decades, pharmacotherapies have been used for OP through either antiresorptive or anabolic means. However, the clinical effects are unsatisfactory due to their prevention of just bone resorption without restoring bone loss or their complex clinical 
treatment protocols[3-5]. Therefore, there is an urgent requirement for innovative effective treatment strategies to promote bone formation in OP patients.

Osteoblasts are the dominant functional cells contributing to bone formation and are derived from bone marrow mesenchymal stem cells (BMSCs). BMSCs give rise to osteoblasts, adipocytes, chondrocytes, and myocytes[6, 7]. Under physiological states, a dynamic balance exists between the osteogenic and the adipogenic potential of BMSCs[8, 9]. Reduced bone formation, but more adipocytes of impaired BMSCs, is one of the main causes of estrogen deficiency and age-related OP[10]. Hence, promoting BMSC osteoblastic differentiation is a promising therapeutic strategy for OP[11]. BMSC differentiation is modulated by various chemical, biological, and physical factors, such as microRNAs. To date, approximately 40 miRNAs have been reported to be involved in the osteogenic differentiation of BMSCs, such as miR-188[12], miR-335-5p[13], miR-210-3p[14], miR-130a[15], miR-149-3p[16], miR-381[17], and miRNA-132-3p[18]. Interestingly, the present study demonstrated that miR-483-5p expression increased in BMSCs from mice with estrogen deficiency and age-related OP. miR-483-5p inhibits the osteogenic differentiation of BMSCs by targeting mitogen-activated protein kinase 1(MAPK1) and SMAD family member 5 (Smad5).

Therefore, antagomir-483-5p (miR-483-5p inhibitor) is expected to become an effective treatment drug for OP. However, the clinical application of novel small molecule nucleic acid drugs has several challenges, such as stimulation of the immune response and poor cellular uptake. Here, we used immunoglobulin $G$ ( $\operatorname{lgG}$ ) and poloxamer-188 (polyoxyethylene-polyoxypropylene block copolymer) to encapsulate antagomir-483-5p[19, 20]. To selectively deliver the nanoparticles to BMSCs, these nanoparticles were conjugated with a BMSC-targeting aptamer[21]. The targeted nanoparticles presented efficient cellular uptake in vitro and promoted bone formation in ovariectomized (OVX) mice, which represent a postmenopausal OP mouse model. Our findings suggested that selectively delivering antagomir-483-5p to BMSCs may represent a novel efficient strategy for the prevention and treatment of OP.

\section{Methods}

\section{OVX-induced experimental OP mouse model}

Eight- to ten-week-old female C57BL/6J WT mice were purchased from Tianqin Biotechnology Co., Ltd., Changsha, China. All mice were maintained under continuous $12 \mathrm{~h}$ light followed by $12 \mathrm{~h}$ dark cycles and pathogen-free conditions. An experimental OP mouse model was induced by bilateral ovariectomy, and control mice were subjected to sham operation. All mice were euthanized for collection of bilateral femur specimens at 12 weeks after surgery. The harvested distal femurs were fixed, decalcified, embedded in paraffin, and cut using our previously established protocol[22]. Routine hematoxylin and eosin (H\&E) staining was used to evaluate bone morphology. All the experimental procedures were approved by the Committees of Animal Ethics and Experimental Safety of Hainan Medical University. 


\section{MicroCT analysis}

The whole secondary spongiosa of the distal femur was scanned ex vivo using a microCT system ( $\mu C T 100$, SCANCO MEDICAL, Switzerland). The trabecular bone was segmented for three-dimensional reconstruction ( $\mu$ CT Evaluation Promgram V6.6, SCANCO MEDICAL, Switzerland) to calculate the following parameters: BV/TV, Conn.D, SMI, trabecular number (Tb.N), Tb.Th, trabecular separation (Tb.Sp), BS/BV and BMD of TV.

\section{Isolation and culture of mouse BMSCs}

For isolation of mouse BMSCs, four- to six-week-old female mice were pretreated in $75 \%$ ethanol solution after the necks were broken for 10-15 min. Bilateral femurs were dissected under sterile conditions. The marrow cavity was repeatedly flushed with $0.0067 \mathrm{M}$ sterile phosphate buffered saline (HyClone, South Logan, UT, USA). The collected bone marrow cells were cultured in Dulbecco's modified Eagle's medium (Gibco, Grand Island, NY, USA) supplemented with 10\% fetal bovine serum (FBS) (Gibco). After $24 \mathrm{~h}$, the cell culture supernatant was discarded, and fresh complete medium was added. When cells reached $85 \%-90 \%$ confluence, $0.25 \%$ trypsin (Gibco) containing $10 \mathrm{mM}$ EDTA was used to digest cells. Only thirdto eighth-passage BMSCs were employed to induce osteogenic and adipogenic differentiation as well as to transfect agomir-483-5p or antagomir-483-5p (GenePharma, Suzhou, China). Lipofectamine 2000 (Invitrogen, Carlsbad, CA, USA) was used for transfection.

\section{RNA extraction and real-time PCR}

Total RNA was extracted from the collected cells using TRIzol reagent (Invitrogen). To analyze $A L P$, Bglap, MAKP1, and Smad5 levels, a Thermo Scientific RevertAid first strand cDNA synthesis kit (Thermo Fisher Scientific, Waltham, MA, USA) was used to synthesize complementary DNAs (cDNAs). The primers (Sangon Biotech, Shanghai, China) used are described in Supplementary Table 1. PowerUP SYBR green master mix (Thermo Fisher Scientific, Waltham, MA, USA) was used to quantify the relative mRNA levels. To analyze miR-483-5p levels, the U6 snRNA real-time PCR normalization kit and the mmu-miR-483-5p hairpin-it real-time PCR kit (GenePharma) were used.

\section{Enzyme-linked immunosorbent assay (ELISA)}

To detect ALP levels in cell culture supernatant, a mouse ALP ELISA kit (AMEKO, Shanghai, China) was used. A mouse Col-1 ELISA kit (AMEKO) was used to measure the concentration of type I collagen in the cell culture supernatant according to the manufacturer's instructions. 


\section{Osteogenesis differentiation and alkaline phosphatase staining.}

To induce osteogenic differentiation of BMSCs, cells were transfected with agomir-483-5p (GenePharma), antagomir-483-5p, or negative control (NC). $6 \mathrm{~h}$ after transfection, cells were cultured with medium containing $10 \mathrm{mM} \beta$-glycerophosphate (Biosharp, Hefei, China) , $1 \mu \mathrm{M}$ dexamethasone (SigmaAldrich, St. Louis, MO, USA), and $50 \mu \mathrm{M}$ ascorbic acid (Solarbio, Beijing, China) for $48 \mathrm{~h}$. Alkaline phosphatase activity was monitored using a BCIP/NBT Alkaline Phosphatase Color Development Kit (Beyotime, Shanghai, China). The lentivirus-mediated miR-483-5p mimics and lentivirus-mediated miR483-5p inhibitor were purchased from GenePharma. For continuous induction, the fresh medium was changed every two days.

\section{Preparation of FAM-BMSC-aptamer-nanoparticles}

First, mouse IgG (Solarbio) was used to package agomir-483-5p or antagomir-483-5p (GenePharma) following the methods described previously ${ }^{19}$. Then, $1 \mathrm{~mL}(400 \mathrm{mM})$ of N-ethyl-N-(3-diethylaminopropyl) carbodiimide (EDC) (Thermo Fisher Scientific) and $1 \mathrm{~mL}(100 \mathrm{mM})$ of N-hydroxysuccinimide (NHS) (Thermo Fisher Scientific) were added to activate terminal carboxyl groups on the surface of nanoparticles. After gentle stirring for $15 \mathrm{~min}$, a total of 5 OD BMSC-targeting-aptamers (5' 6-FAMACGACGGTGATATGCCAAGGTCGTATGCACGAGTCAGAGG-C ${ }_{7}-\mathrm{NH}_{2}-3^{\prime}$ ) (Sangon Biotech) was added, and incubated at room temperature for $2 \mathrm{~h}$ in the dark with gentle shaking. Finally, $0.2 \% \mathrm{v} / \mathrm{v}$ poloxamer- 188 (Sigma-Aldrich, St. Louis, MO, USA) was used to coat FAM-BMSC-aptamer-nanoparticles following methods reported previously ${ }^{19}$.

\section{Characterization of FAM-BMSC-aptamer-nanoparticles}

Particle average hydrodynamic diameters, PDIs, and zeta potentials were measured by a NanoBrook Omni Analyzer (Brookhaven Instruments Corporation, NY, USA). The fluorescence emission spectrum of the FAM-BMSC-aptamer-nanoparticles was determined using a luminal fluorescence spectrometer (Thermo Fisher Scientific). The shape of the nanoparticles was evaluated using a HT-7700 transmission electron microscope (Hitachi, Tokyo, Japan). Biotium's next-generation fluorescent $\mathrm{CF} \circledast$-labeled wheat germ agglutinin ( $\mathrm{CF} \circledast 555 \mathrm{WGA}$ ) was used for plasma membrane imaging on a fluorescence microscope (Olympus, Tokyo, Japan).

\section{Therapeutic inhibition of miR-483-5p in BMSCs of OVX mice.}


Experimental OP model OVX mice were induced as described before. FAM-BMSC-aptamer-nanoparticles carrying agomir-483-5p ( $5 \mathrm{mg} / \mathrm{kg}$ body weight) or antagomir-483-5p ( $5 \mathrm{mg} / \mathrm{kg}$ body weight) were delivered by tail vein injection every week. After 12 weeks, mice were euthanized, and bilateral femur specimens were collected. Left femur specimens were prepared for microCT scans and right femurs were prepared for immunohistochemistry staining.

\section{Cell culture.}

The $\mathrm{C} 3 \mathrm{H} / 10 \mathrm{~T} 1 / 2$, Clone 8 cell line (ATCC, Manassas, VA, USA) was maintained in alpha-modified Minimum Essential Medium (a-MEM, Gibco) containing 10\% FBS (Gibco), 2 mM Lglutamine (Gibco), 1\% MEM non-essential amino acids solution (100X) (Gibco), and $1 \mathrm{mM}$ sodium pyruvate (100 mM) (Solarbio). Cells were maintained under a standard cell culture conditions with $5 \% \mathrm{CO}_{2}$ and $95 \%$ humidity, and they were not used beyond passage 10 .

\section{MAPK1 and Smad53' UTR cloning and Luciferase Assay.}

MAPK1 (gene ID 26413) and Smad5 (gene ID 17129) mRNA 3' UTRs containing the miR-483-5p-binding sequences were amplified by routine PCR. The MAPK1 mRNA 3' UTRs fragment was amplified using the following primers 5'- CCGCTCGAGTCAGAGGACTGGACGAGTTC -3' (forward) and 5'-

AAGGAAAAAAGCGGCCGCAGCCTCCCTACTGTGAAGTG -3' (reverse). The Smad5 mRNA 3' UTRs fragment was amplified using the following primers 5'- CCGCTCGAGACAGTCTCCCTATGCCTGAG -3' (forward) and 5'- AAGGAAAAAAGCGGCCGCTACTCTGCAGTGCATGCCTG -3' (reverse). The PCR products were subcloned into the psiCHECK ${ }^{\mathrm{TM}}$-2 vector (Promega, Madison, WI, USA) through Not I and Xho I (Thermo Fisher Scientific) cloning sites. The binding region mutations were achieved using gene splicing by overlap extension PCR (SOE-PCR). C3H/10T1/2 cells were cotransfected with agomir-483-5p and the constructed plasmid. The dual-luciferase reporter assay system (Promega) was used to measure firefly and renilla luciferase activities on a Spectramax i3x (Molecular Devices, CA, USA).

\section{Inhibition and overexpression of MAPK1 and Smad5}

RNA interference was used to knockdown MAPK1 and Smad5 in BMSCs. siRNA-MAPK1 and siRNASmad5 (Table S2) were synthesized by Sangon Biotech. Overexpression plasmids of the MAPK1 or Smad5 gene were constructed by VectorBuilder. After transfection of the siRNAs or plasmids into cell, the protein levels of MAPK1 and Smad5 were detected by western blot assays. For western blot analysis, rabbit anti-MAKP1 (1:1000) and rabbit anti-Smad5 (1:1000) polyclonal antibodies (ABclonal) were employed as primary antibodies. A HRP-conjugated AffiniPure goat anti-rabbit lgG $(\mathrm{H}+\mathrm{L})$ (Proteintech, IL, USA) was used as the secondary antibody and visualized using an enhanced chemiluminescence kit (Biosharp). 


\section{Immunohistochemistry staining}

After routine deparaffinization and rehydration, all sections were incubated in citrate buffer $(0.01 \mathrm{M}, \mathrm{pH}$ 6.0) at $60^{\circ} \mathrm{C}$ for $16-18 \mathrm{~h}$ for antigen retrieval. Sections were then covered with $3 \%$ hydrogen peroxide for $10 \mathrm{~min}$ in the dark to inactivate endogenous peroxidase activity. Sections were permeabilized and blocked in $1 \%$ goat serum supplemented with $0.2 \%$ Triton X-100 (Beyotime) at room temperature for 30 min. Sections were then incubated overnight at $4{ }^{\circ} \mathrm{C}$ with rabbit anti-MAKP1 (1:100) and rabbit antiSmad5 (1:100) polyclonal antibodies (ABclonal). A HRP-conjugated AffiniPure goat anti-rabbit IgG (H+L) (Proteintech) was used as the secondary antibody and visualized using a DAB detection kit (Origene, Beijing, China).

\section{Statistical analyses}

All numerical data are presented as the mean \pm SEM. All statistical analyses were performed with GraphPad Prism software, version 6.01 (La Jolla, CA, USA). Statistical differences between two groups were determined by the unpaired t test. One-way ANOVA with Dunnett's multiple comparison posttest was used to compare significant differences among multiple groups. All experiments were repeated at least three times, and representative experiments are shown. $P<0.05$ was considered statistically significant ( ${ }^{\star} P<0.05 ;{ }^{\star \star} P<0.01 ; \mathrm{ns}$, nonsignificant).

\section{Results}

\section{miR-483-5p levels are increased in BMSCs of OVX and aged osteoporotic mice}

An experimental model of OP was induced in eight- to ten-week-old female C57BL/6J mice by bilateral ovariectomy. Twelve weeks after surgery, micro computed tomography (microCT) revealed that the trabecular number was reduced in the right femurs of OVX mice (Fig. 1a). BMSCs were isolated from mouse left femurs (Additional file 1: Fig. S1a), and the miR-483-5p levels were significantly increased in OVX mice compared to sham-operated mice (Fig. 1b). Moreover, the mRNA levels of alkaline phosphatase $(A L P)$ and bone gamma-carboxyglutamic acid-containing protein ( $\mathrm{Bglap}$, a gene encoding osteocalcin) in BMSCs were analyzed by real-time PCR. There is a positive correlation between ALP levels and bone formation. Osteocalcin is secreted solely by osteoblasts and is thought to be a marker for the bone formation process. Thus, decreased levels of ALP and Bglap suggested attenuation of BMSC osteoblastic differentiation in OVX mice (Fig. 1c). Compared to young mice (2 months old), the expression of miR-483-5p was increased and the expression of $A L P$ and Bglap was decreased in BMSCs of aged osteoporotic mice (18 months old) (Fig. 1d-f). Taken together, these data confirmed that the levels of miR-483-5p are negatively correlated with BMSC osteoblastic differentiation and OP progression. 


\section{Inhibition of miR-483-5p promotes BMSC osteoblastic differentiation in vitro}

To investigate the role of miR-483-5p in regulating BMSC osteoblastic differentiation, mouse BMSCs were transfected with either agomir-483-5p or antagomir-483-5p. Agomir-483-5p upregulates, and antagomir483-5p downregulates intracellular miR-483-5p levels. Figures $2 a$ and $2 b$ show that the ALP and Bglap mRNA levels were downregulated by agomir-483-5p and upregulated by antagomir-483-5p compared to treatment with the negative control (NC), respectively. Subsequently, the protein concentrations of ALP and collagen I in the cell culture supernatant were assayed by ELISA. Lower levels of collagen I increase the risk of developing OP. Consistently, ALP and collagen I protein concentrations were downregulated by agomir-483-5p and upregulated by antagomir-483-5p compared to the corresponding control treatment (Fig. 2c and 2d).

We next found that agomir-483-5p weakened $\mathrm{ALP}^{+}$staining when osteogenic differentiation was induced in BMSCs for 2 days, whereas antagomir-483-5p enhanced ALP ${ }^{+}$staining (Fig. 2e). In contrast, agomir483-5p enhanced Oil-Red 0 staining when adipogenic differentiation was induced in BMSCs for 2 days, whereas antagomir-483-5p weakened the Oil-Red 0 staining (Additional file 1: Fig. S2a). Furthermore, BMSCs were infected with either lentivirus-mediated miR-483-5p mimics (LV-miR-483-5p mimics) or lentivirus-mediated miR-483-5p inhibitor (LV-miR-483-5p inhibitor) followed by continuous induction of osteogenic differentiation for 21 days. Compared to the corresponding control group, both $A L P$ and Bglap mRNA levels were lower in the LV-miR-483-5p mimics treatment group, while their levels were continuously higher in the LV-miR-483-5p inhibitor group (Fig. $2 f$ and $2 \mathrm{~g}$ ). In addition, we also evaluated the effect of miR-483-5p on peroxisome proliferator-activated receptor (PPAR)- $y$. PPAR- $\gamma$ is essential for adipogenic differentiation in stem cells. PPAR- $\gamma$ mRNA levels were significantly elevated by LV-miR-483$5 p$ mimics but decreased by LV-miR-483-5p inhibitor treatment during continuous induction of adipogenic differentiation in BMSCs for 21 days (Fig. S2b). Taken together, these data demonstrated that inhibition of miR-483-5p promotes BMSC osteoblastic differentiation but reduces adipogenic differentiation.

\section{Preparation and characterization of FAM-BMSC-aptamer- nanoparticles}

A schematic of the BMSC-aptamer-conjugated nanoparticles is shown in Fig. 3a. Briefly, agomir-483-5p or antagomir-483-5p and the corresponding control were packaged with mouse lgG. BMSC-targeting aptamers were then conjugated to the surface of nanoparticles. Finally, to circumvent the reticuloendothelial system during systemic circulation, poloxamer-188 was used to encapsulate the aptamer-conjugated nanoparticles. After optimizing the synthetic route, the spherical shapes of FAMBMSC-aptamer-nanoparticles were observed by transmission electron microscopy (TEM) (Fig. 3b). As shown in Fig. 3c, the average hydrodynamic diameter of FAM-BMSC-aptamer-nanoparticles was $72.30 \pm$ $34.96 \mathrm{~nm}$, which is consistent with the TEM results. In addition, the polydispersity index (PDI) was 0.508 
\pm 0.044 , and the zeta-potential was negative at $9.573 \pm 0.482 \mathrm{mV}$. To confirm the conjugation of FAMBMSC-aptamers, fluorescence microscopy was used to observe the presence of fluorescent green color in the FAM-BMSC-aptamer-nanoparticles. In contrast, there was an absence of fluorescent green color in nonaptamer-conjugated nanoparticles, indicating the lack of FAM-BMSC-aptamer (Fig. 3d). Moreover, the analysis of the fluorescence emission spectrum and fluorescence intensity indicated consistent results (Additional file 1: Fig. S3a).

Fluorescence microscopy was used to ascertain the interaction of FAM-BMSC-aptamer-nanoparticles with the cell membrane of BMSCs. FAM-BMSC-aptamer-nanoparticles (green color) and cells were cocultured for $2 \mathrm{~h}$. The cell membrane was then stained with wheat germ agglutinin (CF®555 WGA) (red color) to image the boundaries of cells. Colocalization of both green and red colors suggested a potential interaction between the FAM-BMSC-aptamer-nanoparticles and the cell membrane (Fig. 3e). After binding to the cell membrane, FAM-BMSC-aptamer-nanoparticles enter the cytosol following the release of agomir-483-5p or antagomir-483-5p. To explore the efficiency of the intracellular release of FAM-BMSCaptamer-nanoparticles, FAM-BMSC-aptamer-nanoparticles and cells were cocultured for $48 \mathrm{~h}$. The mRNA levels of $A L P$ and $B g l a p$ were reduced significantly by FAM-BMSC-aptamer-nanoparticles carrying agomir483-5p (ANPA). In contrast, FAM-BMSC-aptamer-nanoparticles carrying antagomir-483-5p (ANPAn) remarkably promoted the expression of $A L P$ and Bglap (Fig. 3f). In summary, these findings demonstrated that the prepared FAM-BMSC-aptamer-nanoparticles selectively deliver agomir-483-5p or antagomir-483-5p to BMSCs in vitro, thereby regulating the BMSC osteoblastic differentiation.

\section{Therapeutic inhibition of miR-483-5p in OVX mouse BMSCs}

To further test whether therapeutic inhibition of miR-483-5p in BMSCs promotes BMSC osteoblastic differentiation and subsequent bone formation in vivo, OVX mice were administered ANPA or ANPAn via tail vein injection every week. After 12 weeks, the mice were euthanized, and femur BMSCs were isolated. Real-time PCR was used to assay the levels of miR-483-5p in femur BMSCs among the groups. As shown in Fig. 4a, the miR-483-5p levels were increased significantly in ANPA-treated OVX mice compared to the control OVX group. In ANPAn-treated OVX mice, the levels of miR-483-5p were inhibited. These results indicated that the FAM-BMSC-aptamer-nanoparticles can be delivered to BMSCs and functionally regulate the expression of miR-483-5p in vivo.

The trabecular bone of the left distal femur from sham mice, OVX mice, ANPA-treated OVX mice, and ANPAn-treated OVX mice was analyzed by microCT scan (Fig. 4b). In OVX mice, the bone volume fraction (BV/TV), connectivity density (Conn.D), mean/density of TV, and trabecular number (Tb.N) were significantly lower than the sham mice. Previous studies have shown that bones with lower BV/TV, reduced connectivity, decreased mean density, and fewer trabeculae are more susceptible to structural failure and OP. Conn.D and Tb.N are associated with the integrity of trabecular bone microarchitecture in the femur[23]. Excess miR-483-5p in BMSCs (ANPA injection groups) accelerated the reduction in these bone formation-related histomorphometric parameters. Therapeutic inhibition of miR-483-5p within 
BMSCs (ANPAn injection groups) stimulated trabecular bone formation (Fig. 4c-f). There was no difference between OVX and sham mice in the bone formation-related trabecular thickness (Tb.Th). However, inhibition of miR-483-5p rescued the alleviated Tb.Th due to excessive miR-483-5p in BMSCs (Fig. 4g).

By definition, a high bone surface/bone volume (BS/BV) corresponds to bone with a low BV/TV[24]. High BS/BV was observed in ANPA-treated OVX mice, which was recovered to control levels in ANPAn-treated OVX mice (Fig. 4h). Similarly, accompanied by OP progression and increasing miR-483-5p levels in BMSCs, the trabecular separation (Tb.Sp) was increased. In ANPAn-treated OVX mice, the Tb.Sp decreased (Fig. 4i). It is well known that femoral trabeculae with low Tb.Sp, and structural model index (SMI values) indicate plate-like bone microstructure, which highly correlates with elastic modulus and yield strength. In the present study, the effect of miR-483-5p on SMI was not observed (Additional file 1: Fig. S4a).

Taken together, our results confirmed the FAM-BMSC-ptamer-nanoparticles delivered agomir-483-5p or antagomir-483-5p into BMSCs in vivo. Further histomorphometric analysis indicated that inhibition of miR-483-5p levels in BMSCs delays OP progression.

\section{MAPK1 and Smad5 are target genes of miR-483-5p in BMSC osteoblastic differentiation}

To study the molecular mechanism by which miR-483-5p regulates BMSC osteoblast differentiation, the web-based target prediction program, TargetScan, was used to predict the potential targets of miR-483$5 p$, which predicted that MAPK1 and Smad5 are targets of miR-483-5p. Thus, we focused on the MAPK1 and Smad5 genes in the subsequent experiments (Fig. 5a and b). Luciferase assays were used to determine whether MAPK1 and Smad5 are target genes of miR-483-5p. Fig. $5 \mathrm{c}$ and d show that agomir483-5p reduced the luciferase activity of reporters containing the wild-type (WT) MAPK1 and Smad5 3' UTRs but had no effect on reporters with mutated 3'UTRs, which did not bind to miR-483-5p. Although the protein levels of MAPK1 and Smad5 were downregulated by agomir-483-5p treatment and upregulated by antagomir-483-5p treatment, no difference in MAPK1 and Smad5 mRNA levels were observed among the groups (Fig. 5e-h). To determine whether miR-483-5p functionally targets MAPK1 and Smad5 in regulating BMSC osteoblast differentiation, siRNA-MAPK1 (si-MAPK1) and siRNASmad5 (si-Smad5) were employed to knockdown MAPK1 and Smad5 in BMSCs, respectively (Additional file 1: Fig. S5a and b) , and the overexpression efficiency of MAPK1-or Smad5-expressing plasmids was assayed by western blot assay (Additional file 1: Fig. S5c and d). As expected, the mRNA levels of $A L P$ decreased significantly due to agomir-483-5p treatment but were increased by over expression of MAPK1 or Smad5 in BMSCs. Consistently, the antagomir-483-5p-induced high expression of $A L P$ was rescued to normal levels by si-MAPK1 or si-Smad5 (Fig. $5 \mathrm{i}$ and j). Taken together, these results demonstrated that MAPK1 and Smad5 are direct and functional targets of miR-483-5p in BMSC osteoblast differentiation in vitro. 
OVX mice were then established and ANPA or ANPAn were administered through tail intravenous injection every week for 12 weeks. As expected, the MAPK1- or Smad5- positive cells within the femurs of OVX and ANPA-treated OVX mice decreased significantly due to high miR-483-5p levels in BMSCs. ANPAn treatment, which diminished miR-483-5p levels of BMSCs, resulted in increased MAPK1- or Smad5positive cells (Fig. $6 a$ and b). These results suggested that MAPK1 and Smad5 are regulated by miR-483$5 p$ in vivo.

\section{Discussion}

The present study established the important therapeutic role of antagomir-483-5p in OP. Our findings demonstrated that miR-483-5p is a functional molecule important for BMSC osteoblast differentiation and verified that FAM-BMSC-aptamer-nanoparticles carrying antagomir-483-5p are a potential therapeutic means for OP clinically (Fig. 6c).

The miR-483 gene is located in the second intron of the insulin-like growth factor 2 (IGF 2) gene, and the pre-miRNA generates two mature isoforms, namely miR-483-5p and miR-483-3p[25]. miR-483-5p has been reported to be associated with several pathological states, including cartilage-associated disorders such as multiple osteochondroma[26] and osteoarthritis[27, 28], as well as adipose-associated diseases, such as multiple symmetric lipomatosis[29]. In addition, increased levels of miR-483-5p has been identified in OP bone samples[30] and are involved in the pathogenesis by promoting osteoclast differentiation[31]. The present study demonstrated that miR-483-5p inhibits the osteogenic differentiation of BMSCs by targeting the MAPK1 and Smad5 genes. Ye Xiao et al[32] reported that miR-483-3p regulate the osteogenic differentiation of BMSCs by targeting STAT1. Therefore, miR-483 may be a crucial gene in bone- and cartilage- associated metabolism and diseases.

Isoelectric point (pl)-based nanoprecipitation technology[33-35] has been used to prepare nanoparticles. As reported previously, the nanoparticles protect loaded agomir-483-5p or antagomir-483-5p against serum nuclease without inducing the inflammatory response of macrophages while escaping endocytic recycling. BMSC-targeting aptamers were added to the surface of these nanoparticles in our research to ensure directional delivery, resulting in maximized therapeutic efficacy, minimum administered dosage, and minor adverse effects.

However, physicochemical characteristics such as particle size and surface charge of nanoparticles, are more important parameters than their composition[36]. When the particle size is maintained from 50-150 $\mathrm{nm}$, nanoparticles are able to escape macrophage phagocytosis during intravenous administration. Thus, in this study, we optimized the synthetic approaches (simultaneously prolonged stirring time and increased stirring intensity to encapsulate ago-miR-483-5p or antagomir-483-5p with IgG) to produce nanoparticles $72.30 \pm 34.96 \mathrm{~nm}$ in diameter. In addition, nanoparticles with a zeta potential between \pm 10 $\mathrm{mV}$ are cleared in the body bt only approximately $10 \%$. Although the positive charge of nanomedicine promotes integration and endocytosis by cells, it is easily recognized by the reticuloendothelial system in the blood circulation and accelerates the removal efficiency during intravenous administration. Therefore, 
a smaller nanoparticle size and weak negative charge are the basic physical characteristics to improve the therapeutic effect of nanoparticles in vivo. Here, we modulated the concentration of the BMSCtargeting aptamer to produce nanoparticles with a negative value of $9.573 \pm 0.482 \mathrm{mV}$, which resulted in efficient cellular uptake in vitro and in vivo. The optimized targeted drug delivery platform for small molecules will provide new ideas for the treatment of clinical diseases.

Osteogenic differentiation of BMSCs is regulated and induced by many factors, such as MAPK1 (also known as ERK2) and Smad5. In the cytosol, ERK1/2 and Smad1/5 proteins are activated (i.e., phosphorylated) and subsequently translocate into the nucleus where they activate diverse transcription factors to regulate cell survival, metabolism, proliferation, migration, and differentiation. Therefore, it is not surprising that the ERK1/2 and Smad1/5 signaling cascades have been implicated in the pathological conditions of OP and the differentiation of BMSCs. As in previous reports[37-39], knockdown of MAPK1 and Smad5 expression in BMSCs resulted in decreased levels of osteogenicassociated markers, such as ALP, as well as diminished matrix mineralization in vitro and ectopic bone formation in vivo. The present study confirmed that MAPK1 and Smad5 were direct and functional targets of miR-483-5p in BMSC osteoblast differentiation. They are novel targets of miR-483-5p.

\section{Conclusions}

we found that miR-483-5p inhibits BMSC osteoblastic differentiation and that diminishing the miR-483-5p levels in BMSCs is a promising method to control the pathological conditions of OP. To efficiently deliver a miR-483-5p inhibitor (antagomir-483-5p) to BMSCs in vivo, we prepared FAM-BMSC-aptamernanoparticles carrying antagomir-483-5p (ANPAn), and these nanoparticles showed effective OP prevention. Thus, our study provides a novel method for the clinical prevention and treatment of OP.

\section{Abbreviations}

BMSC: bone marrow mesenchymal stem cell; OP: osteoporosis; ALP: alkaline phosphatase; Bglap: bone gamma-carboxyglutamic acid-containing protein; MAPK1: mitogen-activated protein kinase 1; Smad5: SMAD family member 5; IgG: immunoglobulin G; poloxamer-188: polyoxyethylene-polyoxypropylene block copolymer; OVX: ovariectomy; NC: negative control; TEM: transmission electron microscopy; PDI: polydispersity index; ANPA: FAM-BMSC-aptamer-nanoparticles carrying agomir-483-5p; ANPAn: FAMBMSC-aptamer-nanoparticles carrying antagomir-483-5p.

\section{Declarations}

\section{Ethics approval and consent to participate}


All the animal experimental procedures were approved by the Committees of Animal Ethics and Experimental Safety of Hainan Medical University.

\section{Consent for publication}

Not applicable.

\section{Availability of data and materials}

Please contact author for data requests.

\section{Competing interests}

The authors declare no competing financial interests.

\section{Funding}

This work was supported by Hainan Province Key Research and Development Project, China (ZDYF2021128), and the National Natural Science Foundation of China $(81760406,82160425)$.

\section{Authors' contributions}

H.W. concived the project and designed the experiments; Y.Z., H.J., A.H, R.L., and X.Z. performed experiments; all authors analyzed data; and H.W. wrote the manuscript.

\section{Acknowledgements}

Not applicable.

\section{References}

1. Prince RL, Lewis JR, Lim WH, Wong G, Wilson KE, Khoo BC, Zhu K, Kiel DP, Schousboe JT. Adding Lateral Spine Imaging for Vertebral Fractures to Densitometric Screening: Improving Ascertainment of Patients at High Risk of Incident Osteoporotic Fractures. J Bone Miner Res. 2019;34(2):282-9.

2. Zhou T, Yang Y, Chen Q, Xie L. Glutamine Metabolism Is Essential for Stemness of Bone Marrow Mesenchymal Stem Cells and Bone Homeostasis. Stem Cells Int. 2019;2019:8928934. 
3. Balani DH, Ono N, Kronenberg HM. Parathyroid hormone regulates fates of murine osteoblast precursors in vivo. J Clin Invest. 2017;127(9):3327-38.

4. Fan Y, Hanai JI, Le PT, Bi R, Maridas D, DeMambro V, Figueroa CA, Kir S, Zhou X, Mannstadt M, et al. Parathyroid Hormone Directs Bone Marrow Mesenchymal Cell Fate. Cell Metab. 2017;25(3):661-72.

5. Kraenzlin ME, Meier C. Parathyroid hormone analogues in the treatment of osteoporosis. Nat Rev Endocrinol. 2011;7(11):647-56.

6. Yang A, Yu C, Lu Q, Li H, Li Z, He C. Mechanism of Action of Icariin in Bone Marrow Mesenchymal Stem Cells. Stem Cells Int. 2019;2019:5747298.

7. Yang TL, Shen H, Liu A, Dong SS, Zhang L, Deng FY, Zhao Q, Deng HW. A road map for understanding molecular and genetic determinants of osteoporosis. Nat Rev Endocrinol. 2020;16(2):91-103.

8. Kokabu S, Lowery JW, Jimi E. Cell Fate and Differentiation of Bone Marrow Mesenchymal Stem Cells. Stem Cells Int. 2016;2016:3753581.

9. Yang A, Yu C, You F, He C, Li Z. Mechanisms of Zuogui Pill in Treating Osteoporosis: Perspective from Bone Marrow Mesenchymal Stem Cells. Evid Based Complement Alternat Med. 2018;2018:3717391.

10. Wang C, Meng H, Wang X, Zhao C, Peng J, Wang Y. Differentiation of Bone Marrow Mesenchymal Stem Cells in Osteoblasts and Adipocytes and its Role in Treatment of Osteoporosis. Med Sci Monit. 2016;22:226-33.

11. Jin Z, Chen J, Shu B, Xiao Y, Tang D. Bone mesenchymal stem cell therapy for ovariectomized osteoporotic rats: a systematic review and meta-analysis. BMC Musculoskelet Disord. 2019;20(1):556.

12. Li CJ, Cheng P, Liang MK, Chen YS, Lu Q, Wang JY, Xia ZY, Zhou HD, Cao X, Xie H, et al. MicroRNA188 regulates age-related switch between osteoblast and adipocyte differentiation. J Clin Invest. 2015;125(4):1509-22.

13. Zhang L, Tang Y, Zhu X, Tu T, Sui L, Han Q, Yu L, Meng S, Zheng L, Valverde P, et al. Overexpression of MiR-335-5p Promotes Bone Formation and Regeneration in Mice. J Bone Miner Res. 2017;32(12):2466-75.

14. Hu B, Li Y, Wang M, Zhu Y, Zhou Y, Sui B, Tan Y, Ning Y, Wang J, He J, et al. Functional reconstruction of critical-sized load-bearing bone defects using a Sclerostin-targeting miR-210-3p-based construct to enhance osteogenic activity. Acta Biomater. 2018;76:275-82.

15. Lin Z, He H, Wang M, Liang J. MicroRNA-130a controls bone marrow mesenchymal stem cell differentiation towards the osteoblastic and adipogenic fate. Cell Prolif. 2019;52(6):e12688.

16. Li Y, Yang F, Gao M, Gong R, Jin M, Liu T, Sun Y, Fu Y, Huang Q, Zhang W, et al. miR-149-3p Regulates the Switch between Adipogenic and Osteogenic Differentiation of BMSCs by Targeting FTO. Mol Ther Nucleic Acids. 2019;17:590-600.

17. Long H, Zhu Y, Lin Z, Wan J, Cheng L, Zeng M, Tang Y, Zhao R. miR-381 modulates human bone mesenchymal stromal cells (BMSCs) osteogenesis via suppressing Wnt signaling pathway during atrophic nonunion development. Cell Death Dis. 2019;10(7):470. 
18. Hu Z, Zhang L, Wang H, Wang Y, Tan Y, Dang L, Wang K, Sun Z, Li G, Cao X, et al. Targeted silencing of miRNA-132-3p expression rescues disuse osteopenia by promoting mesenchymal stem cell osteogenic differentiation and osteogenesis in mice. Stem Cell Res Ther. 2020;11(1):58.

19. Perepelyuk M, Maher C, Lakshmikuttyamma A, Shoyele SA. Aptamer-hybrid nanoparticle bioconjugate efficiently delivers miRNA-29b to non-small-cell lung cancer cells and inhibits growth by downregulating essential oncoproteins. Int J Nanomedicine. 2016;11:3533-44.

20. Sacko K, Thangavel K, Shoyele SA. Codelivery of Genistein and miRNA-29b to A549 Cells Using Aptamer-Hybrid Nanoparticle Bioconjugates. Nanomaterials (Basel) 2019, 9(7).

21. Luo ZW, Li FX, Liu YW, Rao SS, Yin H, Huang J, Chen CY, Hu Y, Zhang Y, Tan YJ, et al. Aptamerfunctionalized exosomes from bone marrow stromal cells target bone to promote bone regeneration. Nanoscale. 2019;11(43):20884-92.

22. Wang $H$, Zhang $H$, Fan $K$, Zhang D, Hu A, Zeng X, Liu YL, Tan G, Wang H: Frugoside delays osteoarthritis progression via inhibiting miR-155-modulated synovial macrophage M1 polarisation. Rheumatology (Oxford) 2021.

23. Chanpaisaeng K, Reyes Fernandez PC, Fleet JC. Dietary calcium intake and genetics have sitespecific effects on peak trabecular bone mass and microarchitecture in male mice. Bone. 2019;125:46-53.

24. Arnold EL, Clement J, Rogers KD, Garcia-Castro F, Greenwood C. The use of muCT and fractal dimension for fracture prediction in osteoporotic individuals. J Mech Behav Biomed Mater. 2020;103:103585.

25. Veronese A, Lupini L, Consiglio J, Visone R, Ferracin M, Fornari F, Zanesi N, Alder H, D'Elia G, Gramantieri L, et al: Oncogenic role of miR-483-3p at the IGF2/483 locus. Cancer Res 2010, 70(8):3140-3149.

26. Zuntini M, Salvatore M, Pedrini E, Parra A, Sgariglia F, Magrelli A, Taruscio D, Sangiorgi L. MicroRNA profiling of multiple osteochondromas: identification of disease-specific and normal cartilage signatures. Clin Genet. 2010;78(6):507-16.

27. Hua Wang HZ, Qiuyi S, Wang Y, Yang J, Yang J, Zhang T, Luo S, Wang L, Jiang Yu, Zeng C. Daozhang Cai, Xiaochun Bai: Intra-articular Delivery of Antago-miR-483-5p Inhibits Osteoarthritis by Modulating Matrilin 3 and Tissue Inhibitor of Metalloproteinase 2. Mol Ther. 2017;25(3):13.

28. Wang H, Zhang H, Sun Q, Yang J, Zeng C, Ding C, Cai D, Liu A, Bai X. Chondrocyte mTORC1 activation stimulates miR-483-5p via HDAC4 in osteoarthritis progression. J Cell Physiol. 2019;234(3):2730-40.

29. Chen K, He H, Xie Y, Zhao L, Zhao S, Wan X, Yang W, Mo Z. miR-125a-3p and miR-483-5p promote adipogenesis via suppressing the RhoA/ROCK1/ERK $1 / 2$ pathway in multiple symmetric lipomatosis. Sci Rep. 2015;5:11909.

30. De-Ugarte L, Yoskovitz G, Balcells S, Guerri-Fernandez R, Martinez-Diaz S, Mellibovsky L, Urreizti R, Nogues X, Grinberg D, Garcia-Giralt N, et al. MiRNA profiling of whole trabecular bone: identification of osteoporosis-related changes in MiRNAs in human hip bones. BMC Med Genomics. 2015;8:75. 
31. Li K, Chen S, Cai P, Chen K, Li L, Yang X, Yi J, Luo X, Du Y, Zheng H. MiRNA-483-5p is involved in the pathogenesis of osteoporosis by promoting osteoclast differentiation. Mol Cell Probes. 2020;49:101479.

32. Xiao Y, Guo Q, Jiang TJ, Yuan Y, Yang L, Wang GW, Xiao WF. miR4833p regulates osteogenic differentiation of bone marrow mesenchymal stem cells by targeting STAT1. Mol Med Rep. 2019;20(5):4558-66.

33. Dim N, Perepelyuk M, Gomes O, Thangavel C, Liu Y, Den R, Lakshmikuttyamma A, Shoyele SA. Novel targeted siRNA-loaded hybrid nanoparticles: preparation, characterization and in vitro evaluation. $\mathrm{J}$ Nanobiotechnology. 2015;13:61.

34. Lakshmikuttyamma A, Sun Y, Lu B, Undieh AS, Shoyele SA. Stable and efficient transfection of siRNA for mutated KRAS silencing using novel hybrid nanoparticles. Mol Pharm. 2014;11(12):4415-24.

35. Srinivasan AR, Shoyele SA. Self-associated submicron IgG1 particles for pulmonary delivery: effects of non-ionic surfactants on size, shape, stability, and aerosol performance. AAPS PharmSciTech. 2013;14(1):200-10.

36. He C, Hu Y, Yin L, Tang C, Yin C. Effects of particle size and surface charge on cellular uptake and biodistribution of polymeric nanoparticles. Biomaterials. 2010;31(13):3657-66.

37. He F, Ni N, Zeng Z, Wu D, Feng Y, Li AJ, Luu B, Li AF, Qin K, Wang E, et al. FAMSi: A Synthetic Biology Approach to the Fast Assembly of Multiplex siRNAs for Silencing Gene Expression in Mammalian Cells. Mol Ther Nucleic Acids. 2020;22:885-99.

38. Lu N, Malemud CJ. Extracellular Signal-Regulated Kinase: A Regulator of Cell Growth, Inflammation, Chondrocyte and Bone Cell Receptor-Mediated Gene Expression. Int J Mol Sci 2019, 20(15).

39. Wang J, Wang M, Chen F, Wei Y, Chen X, Zhou Y, Yang X, Zhu X, Tu C, Zhang X. Nano-Hydroxyapatite Coating Promotes Porous Calcium Phosphate Ceramic-Induced Osteogenesis Via BMP/Smad Signaling Pathway. Int J Nanomedicine. 2019;14:7987-8000.

\section{Figures}


a

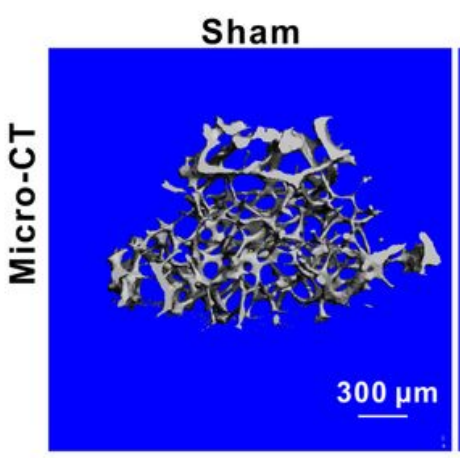

d

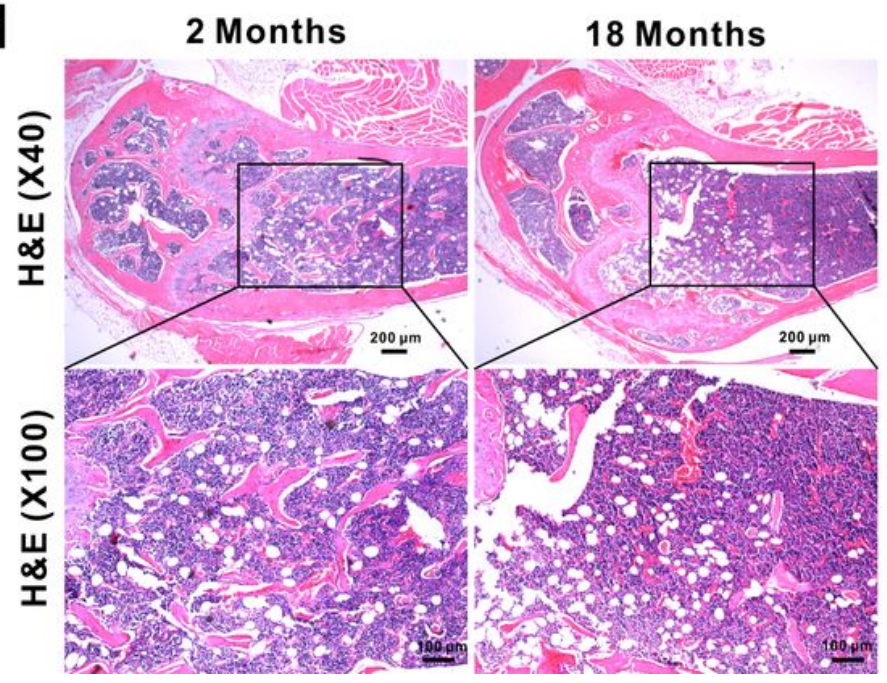

b

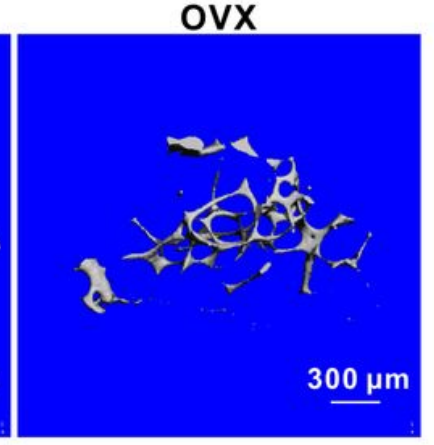

18 Months

e
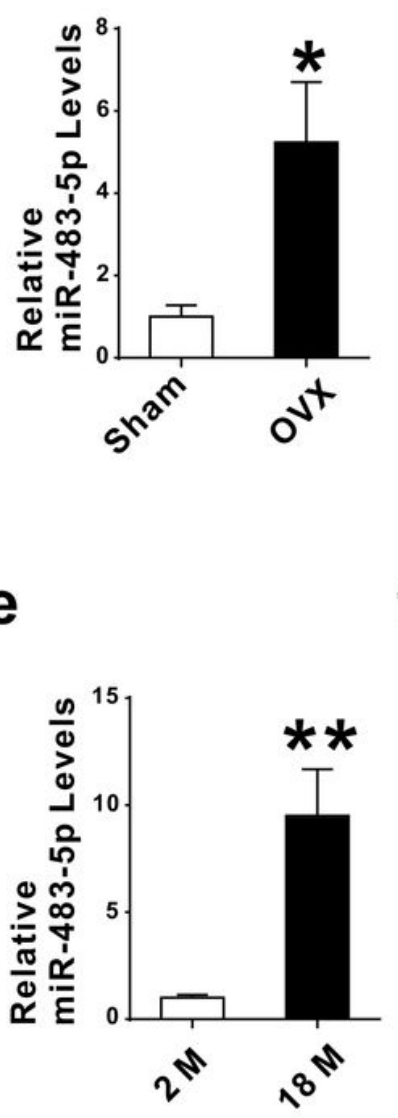

C

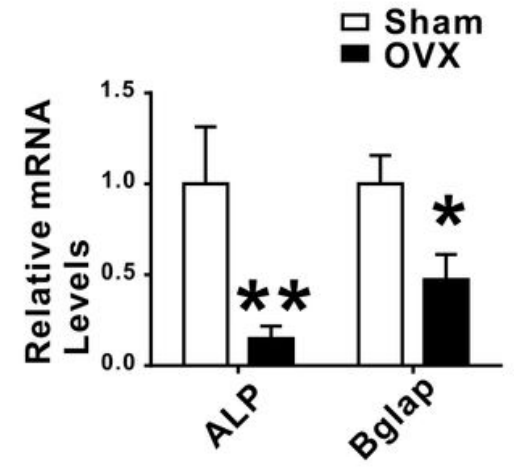

f

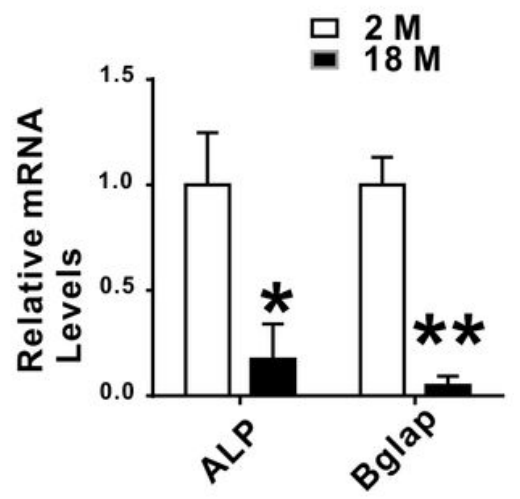

Figure 1

miR-483-5p levels are increased in BMSCs of OVX and aged osteoporotic mice. a MicroCT scan revealed that the trabecular number was reduced in OVX mice compared to sham mice $(n=3)$. b Real-time PCR assay showed a significant increase in the levels of miR-483-5p in BMSCs from OVX mice compared to those from sham surgery mice. $c$ The mRNA levels of ALP and Bglap in BMSCs were analyzed by realtime RCR. $d$ H\&E staining of young or old mouse femur specimens $(n=3)$. e, $f$ Compared to young mice (2 months old) mice, the expression of miR-483-5p was increased and the ALP and Bglap expression was decreased in BMSCs from aged osteoporotic mice (18 months old) osteoporotic mice. H\&E: hematoxylin and eosin. Data are shown as mean $\pm \mathrm{SEM} ;{ }^{*} \mathrm{P}<0.05$, ${ }^{*} \mathrm{P}<0.01$. 
a
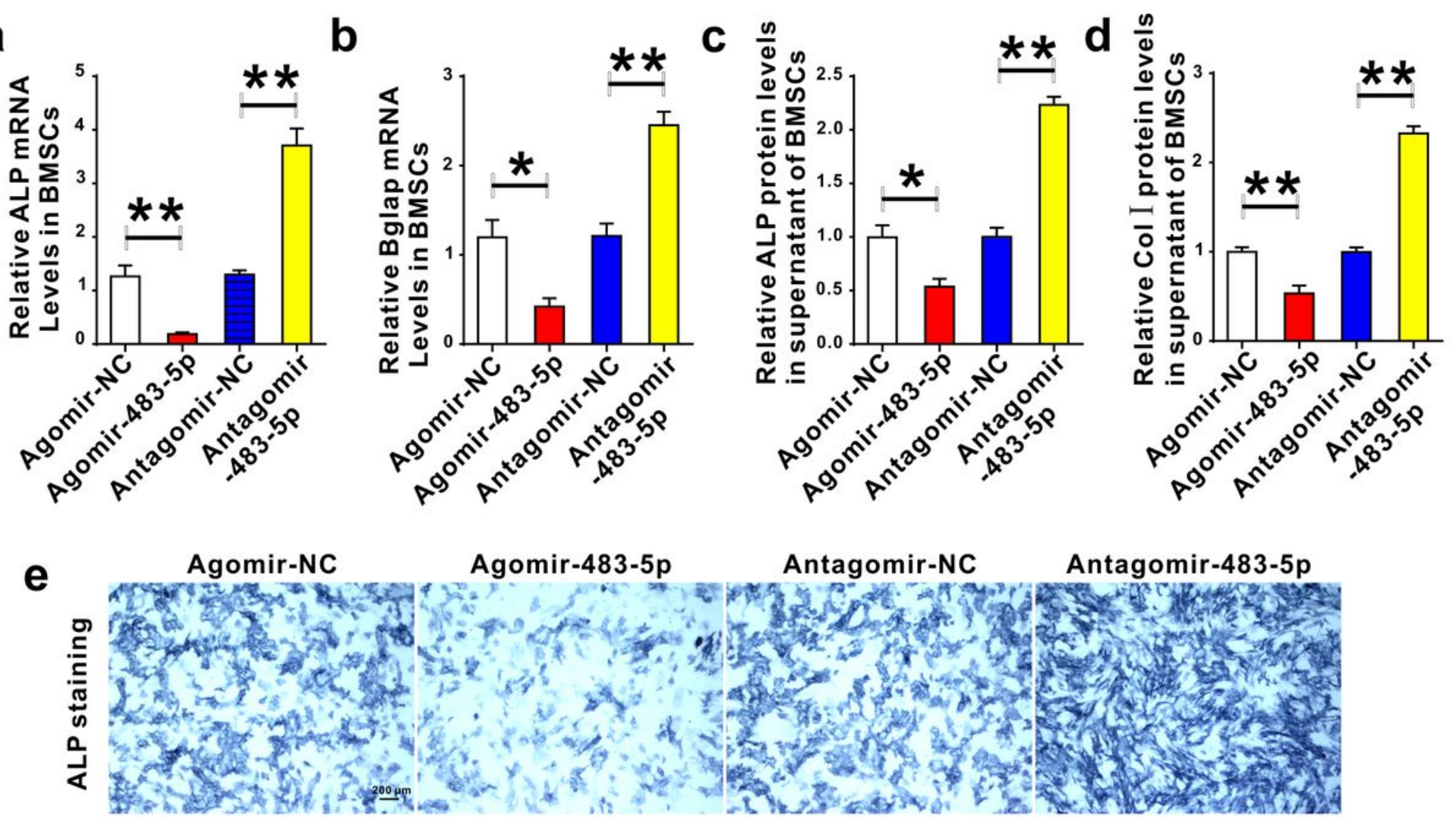

f
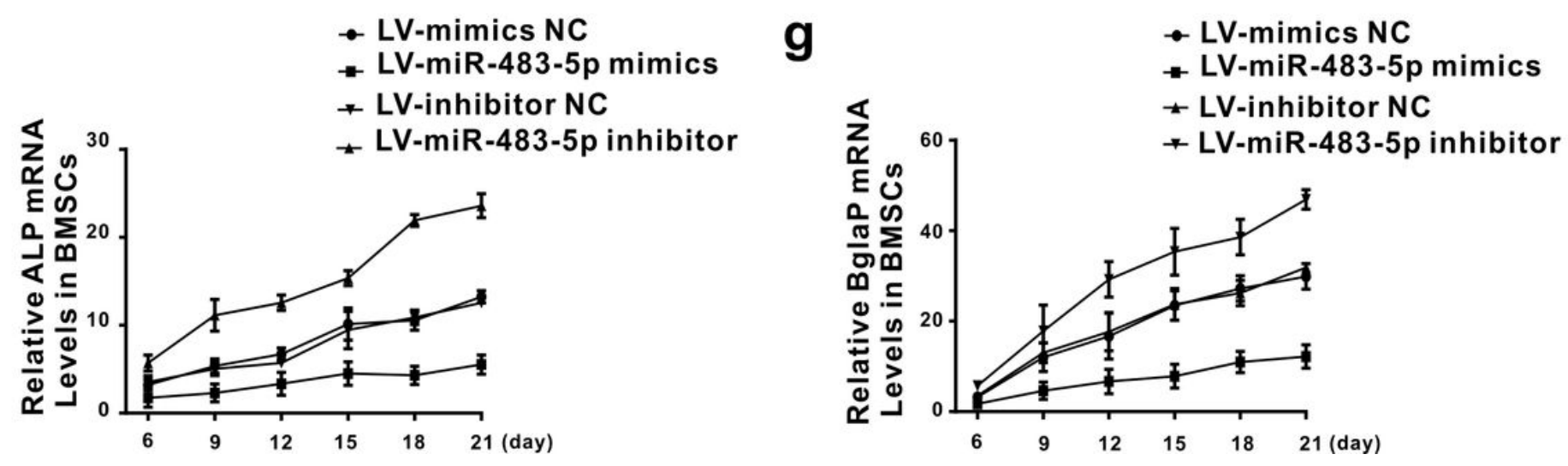

Figure 2

Inhibition of miR-483-5p promotes BMSC osteoblastic differentiation in vitro. a, b Real-time PCR assays showed that ALP and Bglap mRNA levels in BMSCs were downregulated by agomir-483-5p and upregulated by antagomir-483-5p compared to treatment with NC of agomir and antagomir respectively. $c, d$ The protein concentrations of ALP and collagen I in the cell culture supernatant were assayed by ELISA. e BMSCs were induced to undergo osteogenic differentiation for 2 days, and alkaline phosphatase activity was monitored using an alkaline phosphatase assay kit. $\mathrm{f}, \mathrm{g}$ Real-time PCR assay of the ALP and Bglap mRNA levels in BMSCs infected with either LV-miR-483-5p mimics or LV-miR-483-5p inhibitor for 21 days. All data are shown as mean $\pm S E M ; * P<0.05, * * P<0.01$. 
a

FAM-BMSC-aptamer

-nanoparticle

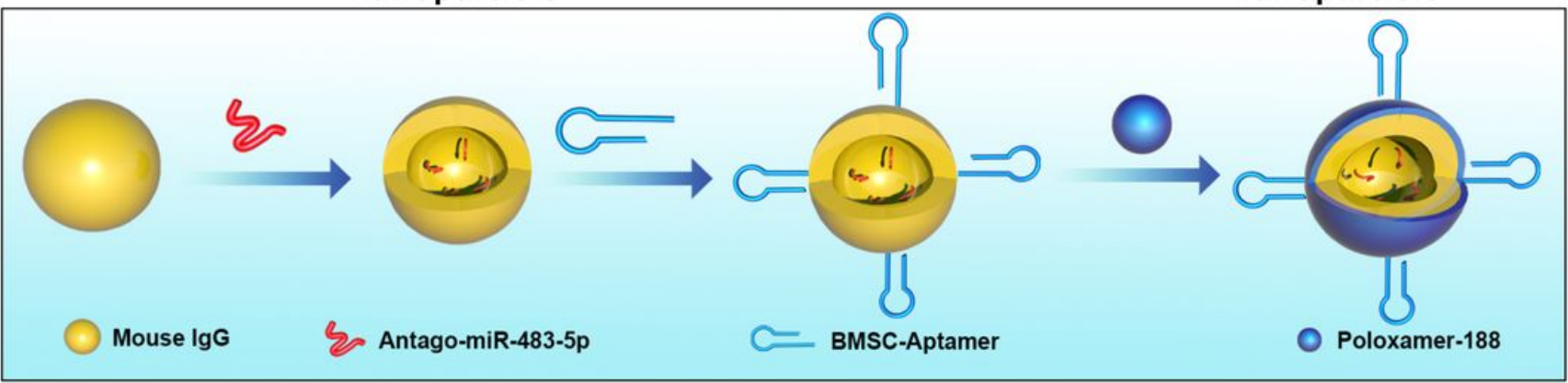

b

TEM

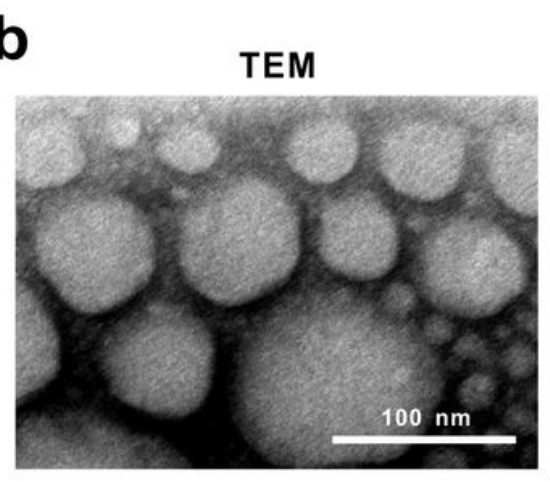

e

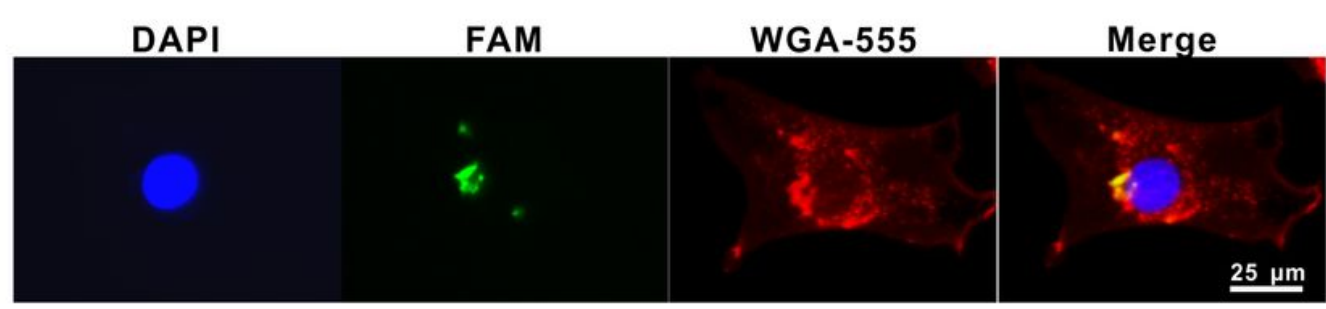

C

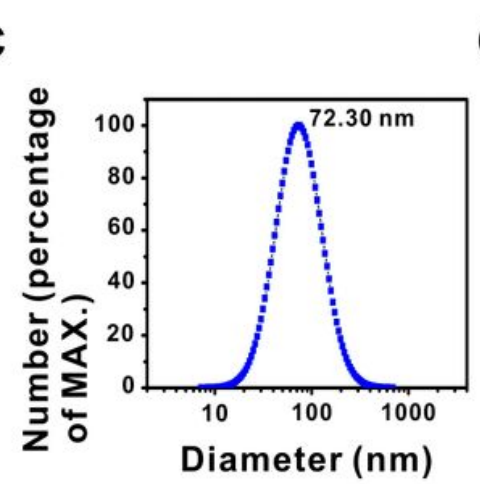

d

$5 \mu \mathrm{m}$
FAM-BMSC-aptamer -nanoparticles

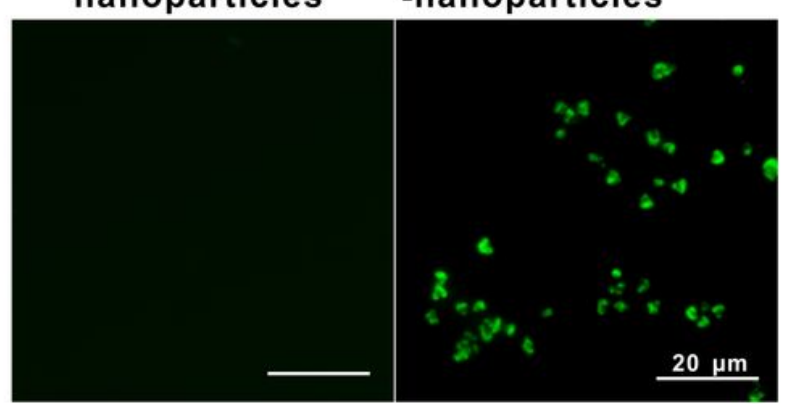

f

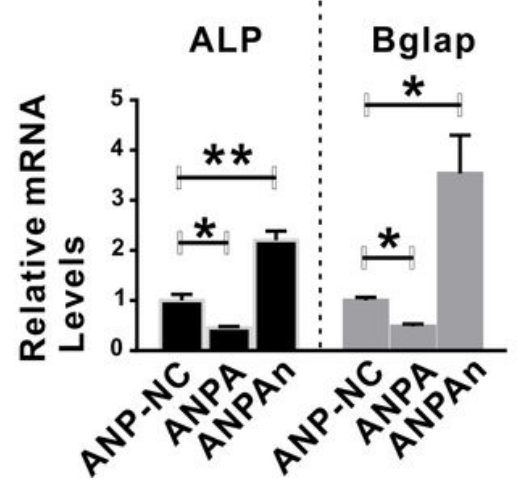

Figure 3

Preparation and characterization of FAM-BMSC-aptamer-nanoparticles. a Schematic of the preparation of BMSC-aptamer-conjugated nanoparticles. b Spherical shapes of FAM-BMSC-aptamer-nanoparticles were observed by TEM. c The size distribution of FAM-BMSC-aptamer-nanoparticles was measured by dynamic light scattering (DLS). d Fluorescence microscopy was used to observe the presence of fluorescent green color in the FAM-BMSC-aptamer-nanoparticles. e Colocalization of both green and red colors suggested a possible interaction between the FAM-BMSC-aptamer-nanoparticles (green) and the cell membrane (red). $f$ After FAM-BMSC-aptamer-nanoparticles and cells were cocultured for $48 \mathrm{~h}$, the mRNA levels of ALP and Bglap were detected by real-time PCR assay. All data are shown as mean \pm SEM; $* \mathrm{P}<0.05, * * \mathrm{P}<0.01$. 
a

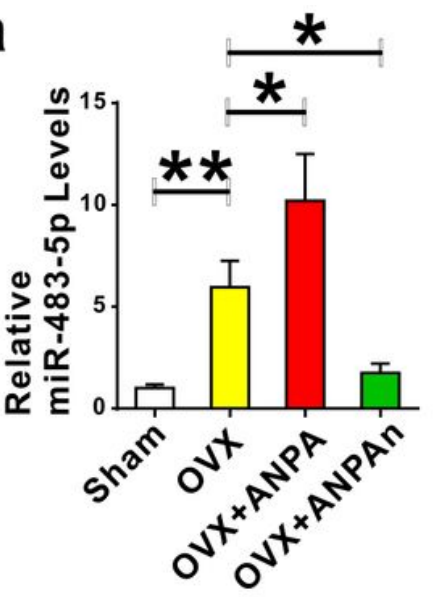

b

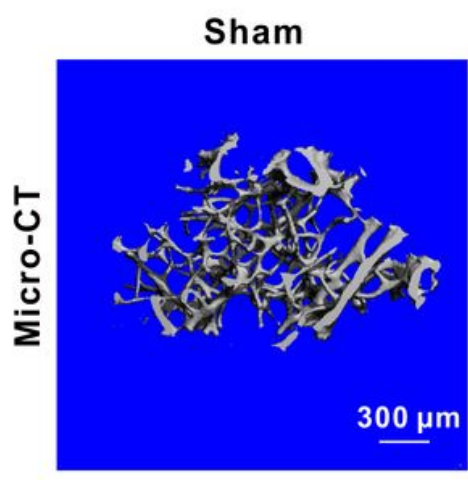

C

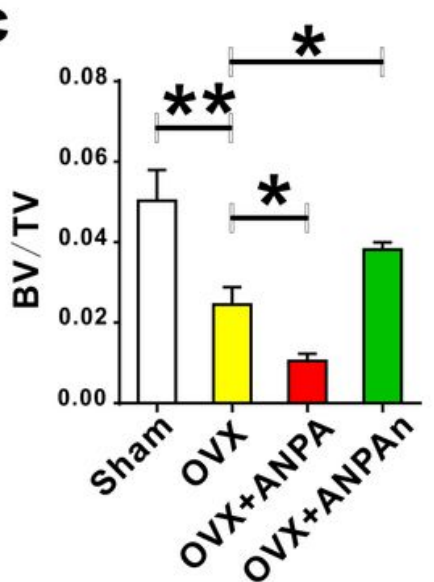

d
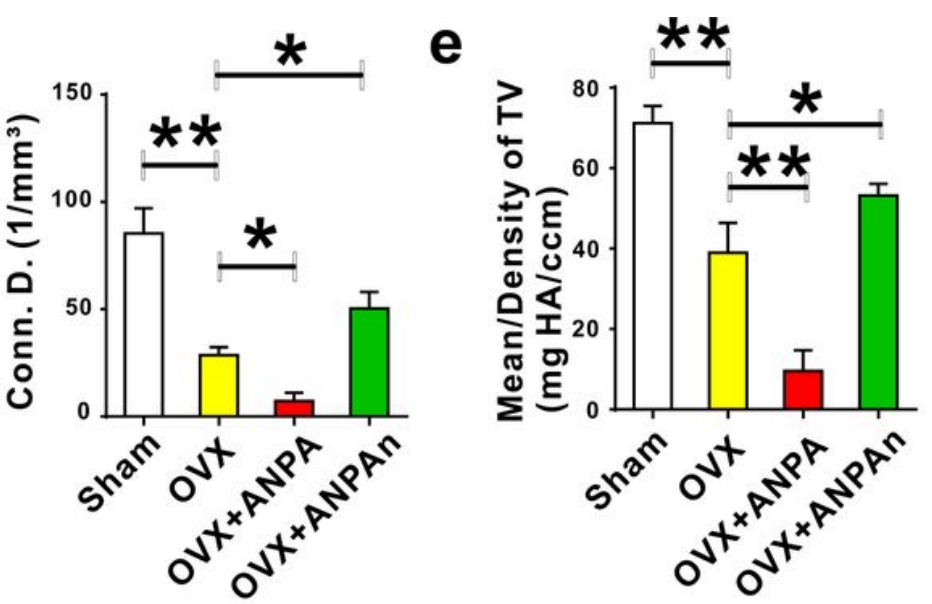

f

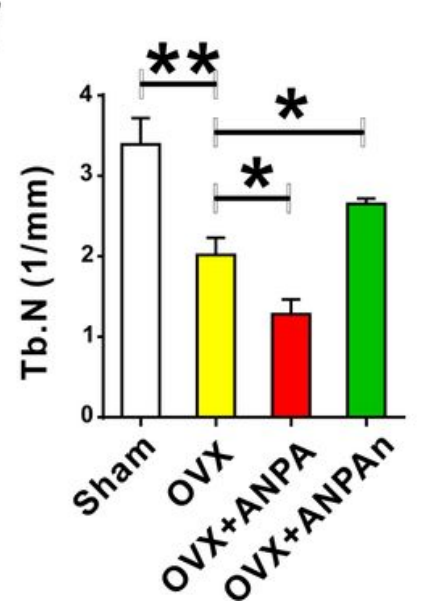

g
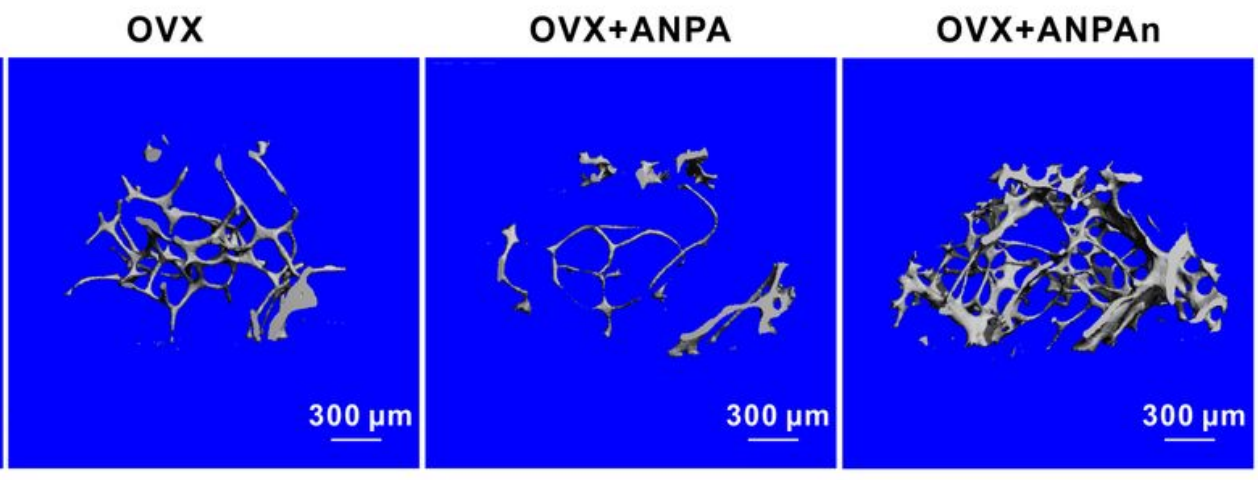

Figure 4

h
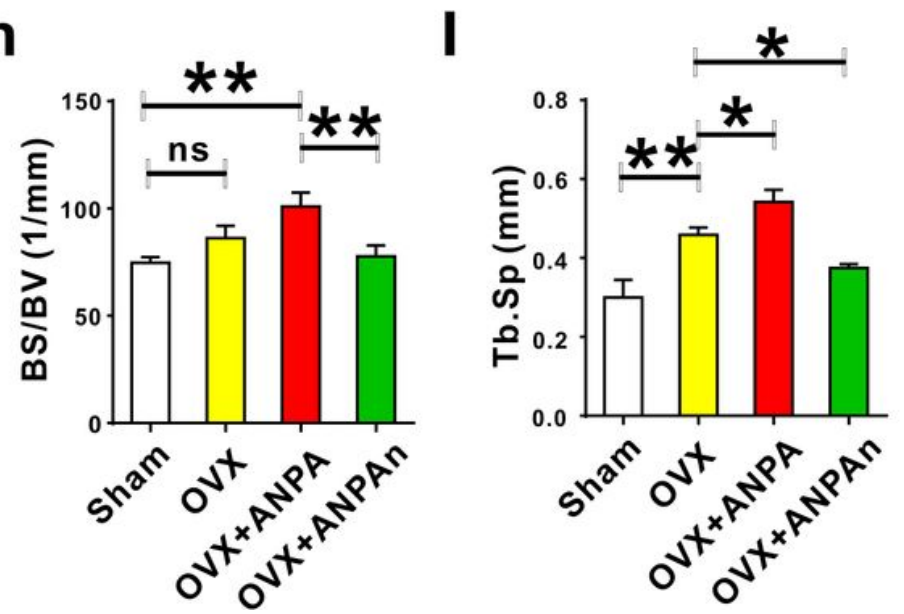

Therapeutic inhibition of miR-483-5p in BMSCs of OVX mice. a Real-time PCR was used to assay the levels of miR-483-5p in femur BMSCs among OVX mice $(n=3)$ administered ANPA or ANPAn by tail vein injection every week for 12 weeks. $b$ The trabecular bone of the left distal femur from sham, OVX, ANPAtreated OVX, and ANPAn-treated OVX mice was analyzed by microCT scan $(n=7)$. c-i Statistical analysis of BV/TV (c), Conn.D (d), mean/density of TV (e), trabecular number Tb.N (f), Tb.Th (g), BS/BV (h), and Tb.Sp (i) based on mice described in (b). All data are shown as mean $\pm S E M ; * P<0.05,{ }^{*} P<0.01$, ns, nonsignificant. 
a

Mmu-miR-483-5p 3' GAGGGAaGAGAaGA GGGCAGA A Wt MAPK1 3' UTR 5' GGUCCUGUCUUCAG CCCGUCU C Mu MAPK1 3' UTR 5' GGUCCUGUCUUCAG GGGCAGA C

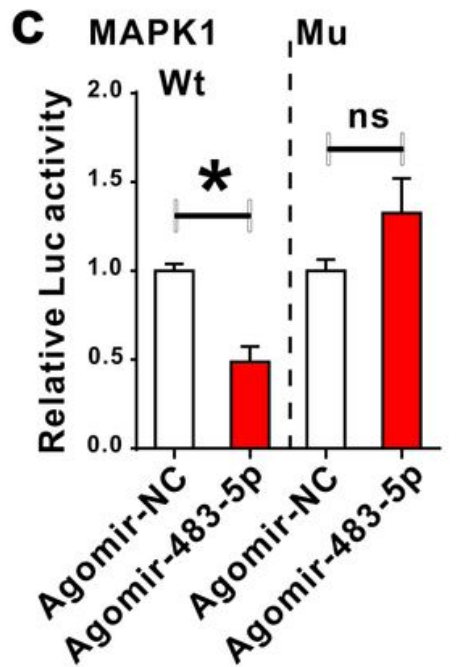

f
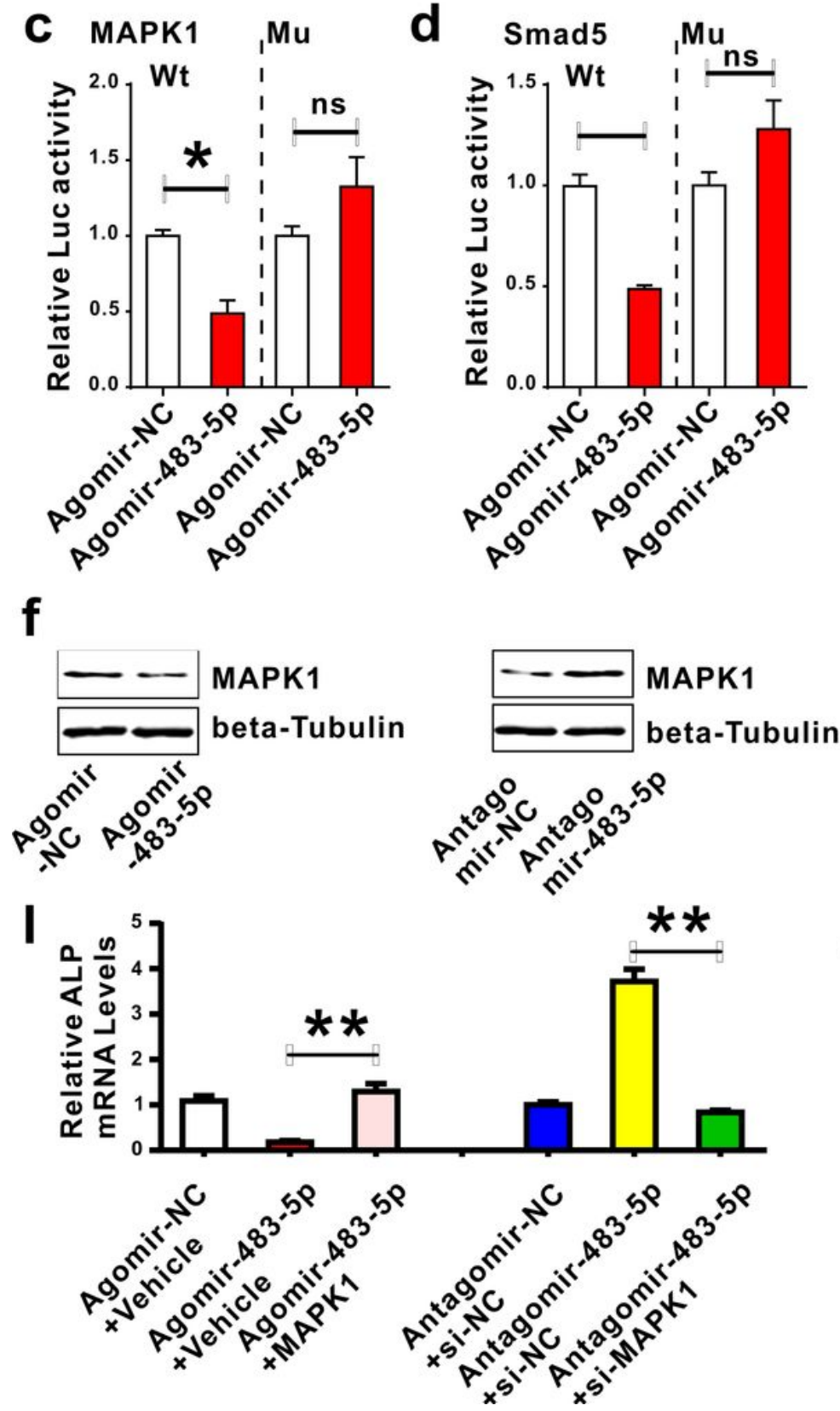

b

Mmu-miR-483-5p 3' GAGGGAAGAGAAG AGGGCAGA A

Wt Smad5 3' UTR 5' GGUCUCUUAAAAG UCCCGUCU C Mu Smad5 3' UTR 5' GGUCUCUUAAAAG AGGGCAGA C
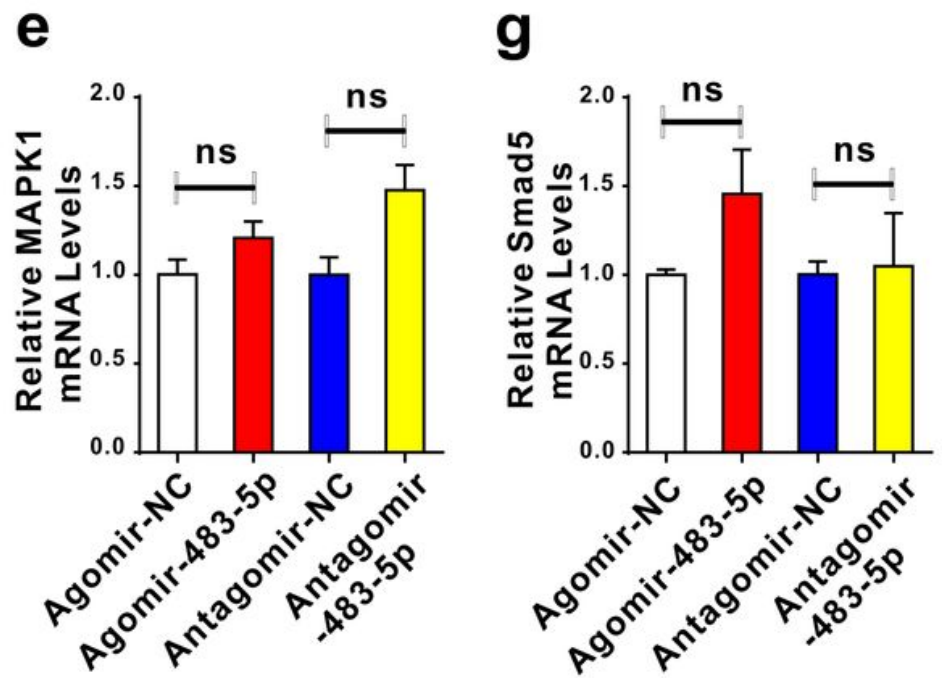

h
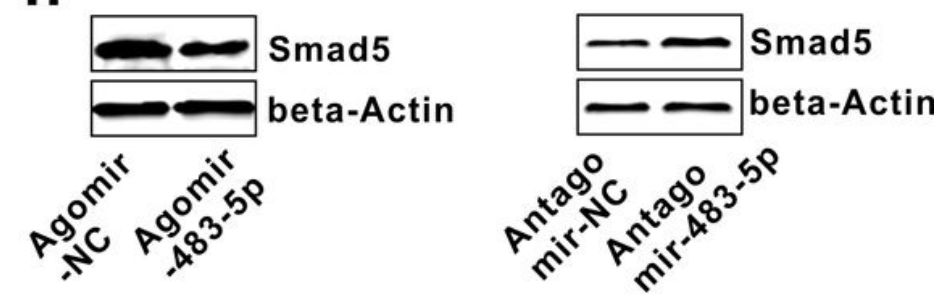
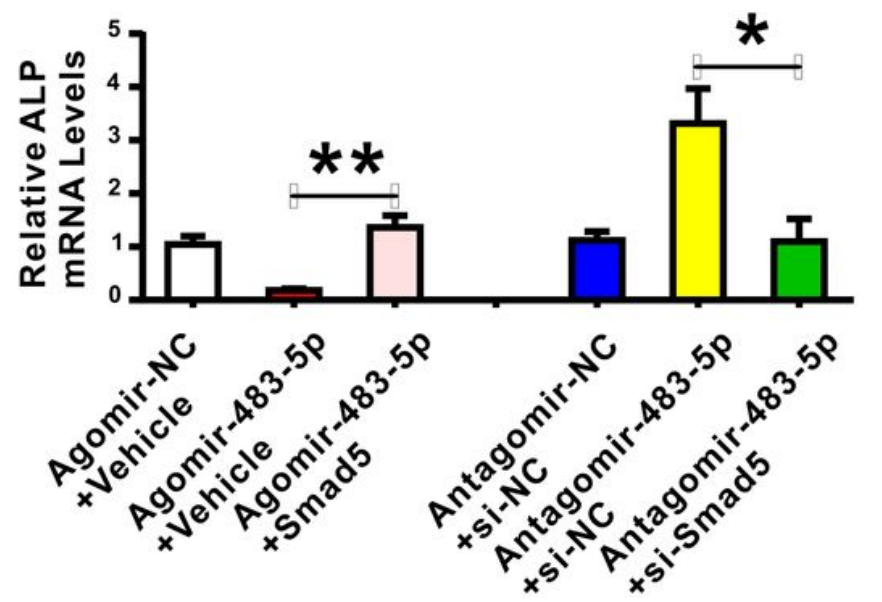

Figure 5

MAPK1 and Smad5 are target genes of miR-483-5p during BMSC osteoblastic differentiation. $a, b$ The web-based target prediction program, TargetScan, predicted MAPK1 and Smad5 as potential targets of miR-483-5p. c, d Luciferase assays. e-h Western blotting was used to detect the protein levels of MAPK1 and Smad5 in BMSCs transfected with agomir-483-5p or antagomir-483-5p for $48 \mathrm{~h}$, and real-time PCR was used to assay MAPK1 and Smad5 mRNA levels among groups. i, j Rescue experiments confirmed that MAPK1 and Smad5 are direct and functional targets of miR-483-5p in BMSC osteoblast differentiation in vitro. All data are shown as mean $\pm \mathrm{SEM}$; ${ }^{*} \mathrm{P}<0.05$, ${ }^{\star *} \mathrm{P}<0.01$, ns, non-significant. 

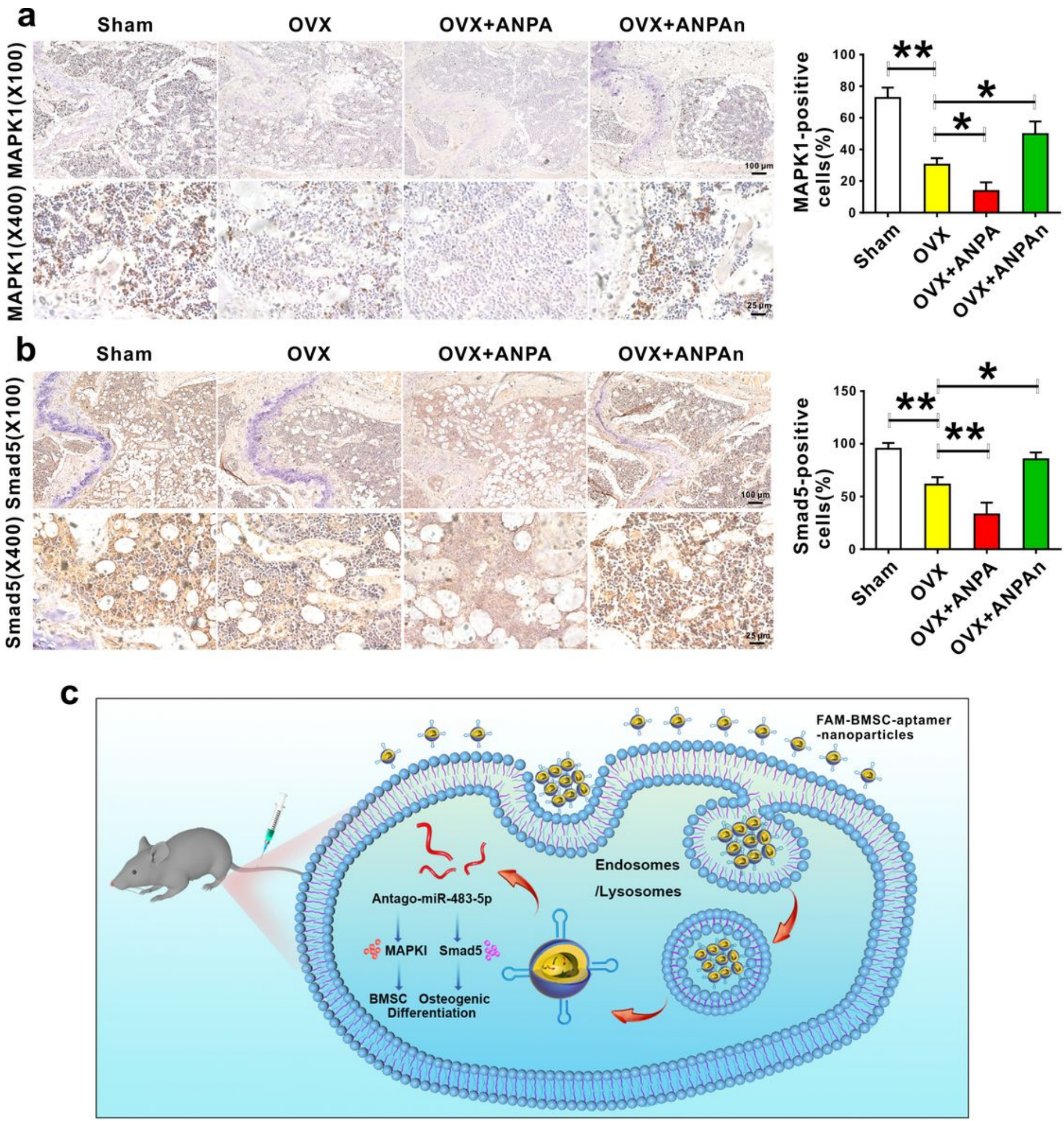

Figure 6

MAPK1 and Smad5 are functional targets of miR-483-5p in vivo. a, b Immunohistochemistry staining was used to analyze MAPK1- or Smad5- positive cells in femurs from sham, OVX, ANPA-treated OVX, and ANPAn-treated OVX mice $(n=7)$. c Graphical representation illustrating the therapeutic pathway of FAMBMSC-aptamer-nanoparticles carrying antagomir-483-5p in controlling the pathological conditions of osteoporosis. All data are shown as mean $\pm \mathrm{SEM}$; ${ }^{*} \mathrm{P}<0.05$, ** $\mathrm{P}<0.01$. 


\section{Supplementary Files}

This is a list of supplementary files associated with this preprint. Click to download.

- Additionalfile1.pdf 